This document is confidential and is proprietary to the American Chemical Society and its authors. Do not copy or disclose without written permission. If you have received this item in error, notify the sender and delete all copies.

\title{
The role of impurities on the kinetic persistence of amorphous calcium carbonate: a nanoscopic dynamics view
}

\begin{tabular}{|r|l|}
\hline Journal: & Journal of the American Chemical Society \\
\hline Manuscript ID & Draft \\
\hline Manuscript Type: & Article \\
\hline Date Submitted by the Author: & n/a \\
\hline Complete List of Authors: & $\begin{array}{l}\text { Koishi, Ayumi; Univ. Grenoble Alpes, ISTerre } \\
\text { Fernandez-Martinez, Alejandro; CNRS, ISTerre } \\
\text { Ruta, Beatrice; European Synchrotron Radiation Facility, } \\
\text { Jimenez-Ruiz, Monica; Institut Laue-Langevin, } \\
\text { Poloni, Roberta ; Laboratoire de Science et Ingénierie des Matériaux et } \\
\text { Procédés (SIMaP), } \\
\text { Di Tommaso, Devis; queen Mary, University of London, School of Biological } \\
\text { and Chemical Sciences } \\
\text { Zontone, Federico; ESRF } \\
\text { Waychunas, Glenn; Lawrence Berkeley National Laboratory, Earth Sciences } \\
\text { Division } \\
\text { MONTES-HERNANDEZ, German; CNRS ISTerre, }\end{array}$ \\
\hline
\end{tabular}

SCHOLARONE

Manuscripts 


\title{
The role of impurities on the kinetic persistence of amorphous calci- um carbonate: a nanoscopic dynamics view
}

\author{
Ayumi Koishi, ${ }^{1}$ Alejandro Fernandez-Martinez, ${ }^{1}$ Beatrice Ruta, ${ }^{2,3}$ Monica Jimenez-Ruiz, ${ }^{4}$ Roberta Po- \\ loni, ${ }^{5}$ Devis di Tommasso, ${ }^{6}$ Federico Zontone, ${ }^{3}$ Glenn A. Waychunas, ${ }^{7}$ German Montes-Hernandez ${ }^{1}$ \\ ${ }^{1}$ Univ. Grenoble Alpes, Univ. Savoie Mont Blanc, XNRS, IRD, IFSTTAR, ISTerre, Grenoble, France \\ ${ }^{2}$ Univ. Lyon, Université Claude Bernard Lyon 1, CNRS, Institut Lumière Matière, Villeurbanne, France \\ ${ }^{3}$ ESRF - The European Synchrotron, Grenoble, France \\ ${ }^{4}$ Institut Laue Langevin, Grenoble, France \\ ${ }^{5}$ SIMAP, CNRS and Univ. Grenoble Alpes, Grenoble, France \\ ${ }^{6}$ Queen Mary University of London, London, UK \\ ${ }^{7}$ Earth and Environmental Sciences Area, Lawrence Berkeley National Laboratory, Berkeley, United States
}

\begin{abstract}
Amorphous calcium carbonate is found in the early stages of biomineral formation. Its moldability and its kinetic persistence against crystallization make it of potential interest to the development of biomimetic materials. The presence of water and of inorganic impurities such as $\mathrm{Mg}^{2+}$ has been reported to increase its kinetic stability. Yet, the precise mechanisms of stabilization as well as the link between structural dynamics and crystallization kinetics are still missing. Here, we report the vibrational and diffusive dynamics of water and other atomic constituents in a series of $\mathrm{Mg}^{2+}$-doped solid amorphous calcium carbonates revealed using coherent X-ray and incoherent neutron scattering. This unique combination of techniques presents a novel view on the nanoscopic dynamic behavior of these amorphous materials: the presence of $\mathrm{Mg}^{2+}$ results in a higher frequency of structural rearrangements within the material. However, this increased dynamic behavior does not translate into a higher crystallization kinetics due to a concomitant stiffening of the water hydrogen bond network.
\end{abstract}

\section{Introduction}

Amorphous calcium carbonate (ACC) is a metastable precursor found during the formation of calcium carbonate biominerals. ${ }^{1-4}$ Calcifying organisms take advantage of the moldable character of ACC and of its exceptional kinetic persistence against crystallization in order to form their shell and skeleton structures, which bear intricate shapes and advanced functionalities. ${ }^{5}$ Given the potential of this amorphous precursor strategy in the field of biomimetics, ${ }^{6}$ particular attention has been devoted to obtaining a mechanistic understanding of the polymorph selection and the kinetic control during the crystallization process from ACC. ${ }^{7,8}$ Previous studies on biogenic ACC have revealed that the stability of the amorphous phase is strongly influenced by the presence of water ${ }^{5}$ and impurities (ranging from co-ions to organic molecules), ${ }^{9-11}$ and interfacial and confinement effects. ${ }^{12,13}$ Among many factors, the role of water, known as key to structural stability $^{14}$, and that of the $\mathrm{Mg}^{2+}$ ion, a common impurity, ${ }^{5,9}$ are the focus of our work.

Some studies have described the crystallization as a dissolution-reprecipitation process on the basis of isotopic tracing experiments ${ }^{15}$ or physico-chemical analyses of crystallization products. ${ }^{16}$ This view is related to the widely accepted understanding that the high dehydration energy of aqueous $\mathrm{Mg}^{2+}$ is a prominent kinetic barrier to calcite nucleation and growth from solution in the presence of $\mathrm{Mg}^{2+} \cdot{ }^{17-19}$ Other studies, however, have reported that transformation of biogenic ACC to calcite during the sea urchin spicule growth proceeds without an identifiable crystallization front. ${ }^{5,20,21}$ This observation is consistent with the view that the crystallization mainly proceeds through solid-state transformations initiated by physisorbed water-mediated partial dissolution. ${ }^{22,23}$

A potentially important clue to the type of crystallization mechanism is the observation that biogenic ACC exists in both hydrous or anhydrous states, with the latter being the transient phase prior to crystallization. ${ }^{2-4,24}$ Previous studies identified different "types" of water in hydrous ACC, typically named as mobile water or rigid structural water, bearing different degrees of restricted motion, of which the gradual loss contributes to the crystallization. ${ }^{25-27}$ An interesting observation made by Reeder et al. is that mobile water is in fact absent in close vicinity of carbonate groups in biogenic ACC from lobster gastrolitsh. ${ }^{28}$ From this, the authors suggest that the mobile water component might facilitate the transport and structural rearrangement of the carbonate framework, which infers that the topological distribution of different types of water may be the key. Reflecting on this, $\mathrm{Mg}^{2+}$ may be able to regulate this structural rearrangement process to a nonnegligible extent by affecting the water exchange dynamics, ${ }^{29,30}$ owning to its high barrier to dehydration as compared to $\mathrm{Ca}^{2+} .31$

Thermodynamic factors provide a basis for the crystallization pathway across a free-energy landscape for any given system. The way the system actually explores this landscape, however, is strongly influenced by kinetic factors such as how rapidly or readily ionic species diffuse and internal cluster 
structures $\operatorname{relax}^{7}$. Despite their relevance to crystallization processes, the experimental elucidation of such dynamic factors during crystallization remains scarcely explored. The foremost goal of the present study, therefore, is to establish a direct link between the dynamics of water molecules and other constituents of ACC and the crystallization kinetics. We examine this link in particular in relation to the role of $\mathrm{Mg}^{2+}$. Given its distinct dehydration energy, we hypothesize that $\mathrm{Mg}^{2+}$ enhances the persistence of ACC via the formation of a strong H-bonding network and, in doing so, creates a barrier to structural rearrangements, thus delaying crystallization.

The dynamic properties of water present in amorphous carbonates were investigated by directly probing the hydrogen bond dynamics using inelastic incoherent neutron scattering. This technique, successfully applied to the studies of hydrogenated materials and confined water ${ }^{32-34}$, is not limited by selection rules as in IR or Raman spectroscopy, and it is highly sensitive to intermolecular interactions of water molecules. To elucidate the structural relaxation dynamics of disordered systems such as amorphous carbonates, a derivative of dynamic light spectroscopy in the X-ray regime, X-ray photon correlation spectroscopy (XPCS), was employed. Thanks to the use of a partially coherent X-ray source and a two-dimensional detector, this technique enables capturing the spatial arrangements of individual scatterers providing a quantitative measure of the rate of collective structural relaxation and information about the nature of such dynamics. XPCS has been successfully used to describe the dynamic behavior of out-of-equilibrium systems such as metallic glasses ${ }^{35}$ or colloidal systems. ${ }^{36}$ It is here used for the first time to probe structural rearrangements within ACC and $\mathrm{Mg}^{2+}$-bearing amorphous carbonates.

\section{Methods}

Sample preparation. A series of solid amorphous carbonates with stoichiometry $\mathrm{Ca}_{(1-x)} \mathrm{M}_{x} \mathrm{CO}_{3} n \mathrm{H}_{2} \mathrm{O}(\mathrm{M}=\mathrm{Mg}, \mathrm{Sr}$ and $\mathrm{Ba}$ ) were synthetized as model systems to biogenic ACC under controlled conditions. A highly basic environment $(\mathrm{pH}$ $\approx 11-13$ ) was employed in order to facilitate the precipitation of stable amorphous carbonates in large quantities, enabling neutron scattering studies. Using the same protocol, a previous study showed that the short range order of this basic ACC is similar to that of biogenic ACC except for the minor presence of hydroxyl groups. ${ }^{28}$ The high $\mathrm{pH}$ synthesis methodology has also been used by other authors to determine the formation enthalpies of ACC and $\mathrm{Mg}^{2+}$-doped ACC, up to amorphous magnesium carbonate (AMC). ${ }^{37-39} \mathrm{~A}$ smooth transition in the short range order from ACC to AMC has been demonstrated by X-ray Pair Distribution Function analyses. ${ }^{38}$

The synthesis procedure involves the rapid mixing of equimolar divalent cation and carbonate solutions (Table S1) followed by immediate quench with acetone and vacuumfiltration. ${ }^{38}$ The mixture typically yields a $\mathrm{pH}$ of $\approx 11$; for pure ACC, this was adjusted to $\approx 13$ with $\mathrm{NaOH}$ to favor the precipitation of an amorphous basic calcium carbonate, yielding a long-term stability. ${ }^{40}$ Prior to the synthesis, all glassware was cleaned with $20 \% \mathrm{HCl}$ to remove any possible nucleation loci and rinsed thoroughly with ultrapure water. Glassware, as well as all the solutions used in the synthesis, were kept in a $4^{\circ} \mathrm{C}$ refrigerator before use to prevent crystallization upon mixing. Vacuum-filtered samples were verified to be amorphous by ATR-FTIR (Nicolet iS10; Supporting Figure S1). These measurements were also used to verify the absence of $\mathrm{Ca}$ and
Mg hydroxide precipitates, which are susceptible to precipitate at highly alkaline conditions. ${ }^{25,26,28,41}$ Once filtered, solid samples were transferred to a dry desiccator and kept until analysis. Additionally, a fraction of each sample was dried at $80^{\circ} \mathrm{C}$ in a vacuum oven for one hour and then kept in a dry desiccator until analysis after the same verification by ATR-FTIR. The chemical compositions of the solid samples ( $x$ values) were obtained by ICP-OES (Varian 720ES) and the water contents ( $n$ values) were determined by thermogravimetric analyses as described later.

Inelastic incoherent neutron scattering (IINS). Hydrogen is a strong spin-incoherent scatterer with a distinguishably large incoherent scattering cross-section (Table S2). Given the relative abundance of ${ }^{1} \mathrm{H}$ relative to ${ }^{2} \mathrm{H}\left(\sim 10^{-4}\right)$, the scattering by hydrogen can be considered mostly incoherent. Therefore, using an appropriate spectrometer, the so-called vibrational density of states of the excitation spectrum can be probed. This last provides precious information on the dynamical properties of the H-bonding network with minimum contributions from non-hydrogen-bearing components $\left(\mathrm{Ca}, \mathrm{CO}_{3}\right.$ and $\mathrm{Mg}$ ). In particular, the excitation energies between 300 and $1200 \mathrm{~cm}^{-1}(\approx 37-148 \mathrm{meV})$ are sensitive to librational modes: intermolecular interactions of water molecules that probe the hindered rotational motions of the H-bonding network and are difficult to explore with other optical probes. ${ }^{42}$

IINS measurements were performed at the Institute LaueLangevin (ILL) in Grenoble, France, using the IN1LAGRANGE (LArge GRaphite ANalyser for Genuine Excitations) secondary spectrometer setup installed at the hot source of the high-flux reactor yielding an energy resolution $\Delta \mathrm{E} / \mathrm{E}$ of $\sim 2-3 \%{ }^{43}$ About $100-200 \mathrm{mg}$ of solid samples were loaded in hollow aluminum sample holders and the data were collected in the range of excitation energy transfer between 21.5 and $195.3 \mathrm{meV}$ at $10 \mathrm{~K}$ in order to decease the Debye-Waller factor. Acquisitions lasted for an average of 5 hours per sample. The obtained intensity was normalized to the monitor counts and corrected for the monitor efficiency using the LAMP package provided by ILL. The background spectrum from the cryostat and an empty sample holder were measured separately and then subtracted from the raw IINS spectrum of the sample. To help with the interpretation of the IINS data, computational simulations were used to produce vibrational density of states (VDOS) for hydrated $\mathrm{Ca}^{2+}$ and $\mathrm{Mg}^{2+}$ as well as monohydrocalcite and compared to the experimentally obtained data (see Supporting Information section 2. Computational simulations methods).

X-ray photon correlation spectroscopy (XPCS). XPCS takes advantage of a high flux coherent X-ray beam to register "speckle patterns" scattered by a disordered system. Thanks to the incoming coherent X-ray, the random diffraction pattern collected on a 2D detector is nothing but the exact spatial arrangement of the particles, or density domains in the probed size-range (see Supporting Figure S2 for representative speckle patterns). Therefore, if the particle positions were to fluctuate, so does the speckle pattern. Their temporal fluctuations in intensity thus provide information on the dynamics of structural rearrangements at the length scale defined by the experimental $q$ range ( $q$ being the modulus of the momentum transfer, $\left.q=|\boldsymbol{q}|=\left|\boldsymbol{k}_{\mathbf{i}}-\boldsymbol{k}_{\boldsymbol{f}}\right|\right)$. This is evaluated by computing the intensity autocorrelation function:

$$
g^{(2)}(\Delta t, q)=\frac{<I(t, q) I(t+\Delta t, q)>}{<I(t, q)>^{2}}
$$


where $\langle\ldots\rangle$ denotes the ensemble average. This function simply provides the correlation between intensities of a given $q$ region within the $2 \mathrm{D}$ detector separated by a lag time $\Delta t$.

Data were collected at the ID10 Troika beamline at the European Synchrotron Radiation Facility (ESRF) in Grenoble, France. Measurements were performed at ambient temperature and under active vacuum ( $\sim 5$ mbar) with an incident energy of $8.1 \mathrm{keV}(\lambda=1.53 \AA)$ in a horizontal scattering geometry over a $q$ range of 0.001 to $0.022 \AA^{-1}$. The coherent part of the incoming X-ray beam $(10 \times 10 \mu \mathrm{m})$ was selected by a pinhole placed right in front of the sample. The solid samples were loaded into steel gaskets with a $100 \mu \mathrm{m}$-wide hole, which was then mounted vertically in the vacuum chamber. $2 \mathrm{D}$ speckle patterns were recorded using a Maxipix photon counting area detector with $256 \times 256$ pixels (pixel size $55 \mu \mathrm{m}$ ). Acquisition times and the number of intensity attenuators were carefully adjusted to avoid beam damage to the samples, which was monitored by plotting two-time correlation functions (representative data shown in Supporting Information). ${ }^{44}$ Accordingly, acquisition times of $0.04 \mathrm{~s}$ were chosen for most of the samples, with a sleeping time of $1.96 \mathrm{~s}$ between images, thus leading to a lag time $\Delta t$ of $2 \mathrm{~s}$ between subsequent speckle patterns. Only sample $x=0.35$ was measured with a higher dose: $0.04 \mathrm{~s}$ acquisition times and $0.46 \mathrm{~s}$ sleeping time $(\Delta t=$ $0.5 \mathrm{~s})$. The profile of the scattered intensity, $I(q)$, was obtained from the same data by performing azimuthal averages of the 2D speckle patterns at different $q$ values. Intensity autocorrelation functions, $g^{(2)}$, were generated using the pyXPCS software provided by the ID10 beamline and fitted at a given $q$ with a Kohlrausch-Williams-Watts (KWW) model:

$$
g^{(2)}(t)=1+c * \exp \left(-2 *(t / \tau)^{\beta}\right)
$$

where $c$ is a set-up dependent contrast, $\tau$ is the structural 'relaxation time', i.e., the characteristic period of time for the system to attain a spatial arrangement different from that of a given initial time point, and $\beta$ is a 'shape factor' referring to the nature of the relaxation processes. An ideal Brownian diffusive system will show an exponent of $\beta=1$. Values of $\beta<$ 1 are typically associated with dynamical heterogeneities in the sample, whereas values $\beta>1$ are associated with stressed or 'jammed' systems. ${ }^{45,46}$

Thermogravimetric and calorimetric analyses. Samples were loaded in alumina crucibles and heated from 25 to $1100^{\circ} \mathrm{C}$ at $15^{\circ} \mathrm{C} / \mathrm{min}$ under argon flow of $20 \mathrm{~mL} / \mathrm{min}$ (Mettler Toledo TGA/DSC3 + ). The water content ( $n$ value) in each sample was quantified from the first weight loss step in the TGA curves and was further distributed into two types of water from the perspective of thermal stability, namely mobile and rigid. The assignment was operatively achieved by a rigorous determination of the first two inflection points found on the second derivatives of the TGA curves (see Supporting Figure S3 for detailed methodology).

\section{Results and Discussion}

Water dynamics. The librational spectra shown in Figure 1 stem from the contributions of rock, twist and wag librational modes of water molecules in pure water and the $\mathrm{Mg}^{2+}$-doped ACC. Water in its liquid state shows a broad distribution of modes (Figure 1a, top) due to the disordered nature of the Hbonding network, while at $10 \mathrm{~K}$ the spectrum displays sharp features due to the formation of crystalline ice and to the degeneration of the librational modes. ${ }^{47}$ Water in $\mathrm{Mg}^{2+}$-doped
ACC (Figure 1a bottom), on the contrary, displays broad features at $10 \mathrm{~K}$. This indicates a distribution of modes (broken degeneracy) suggesting a heterogeneity of local hydrogen environments or structural disorder in the H-bonding network. Interestingly, this feature is proportional to the amount of $\mathrm{Mg}^{2+}$, as is best shown in the decreasing slope of the librational edge between 400 and $600 \mathrm{~cm}^{-1}$ (Figure $1 \mathrm{~b}$ ).

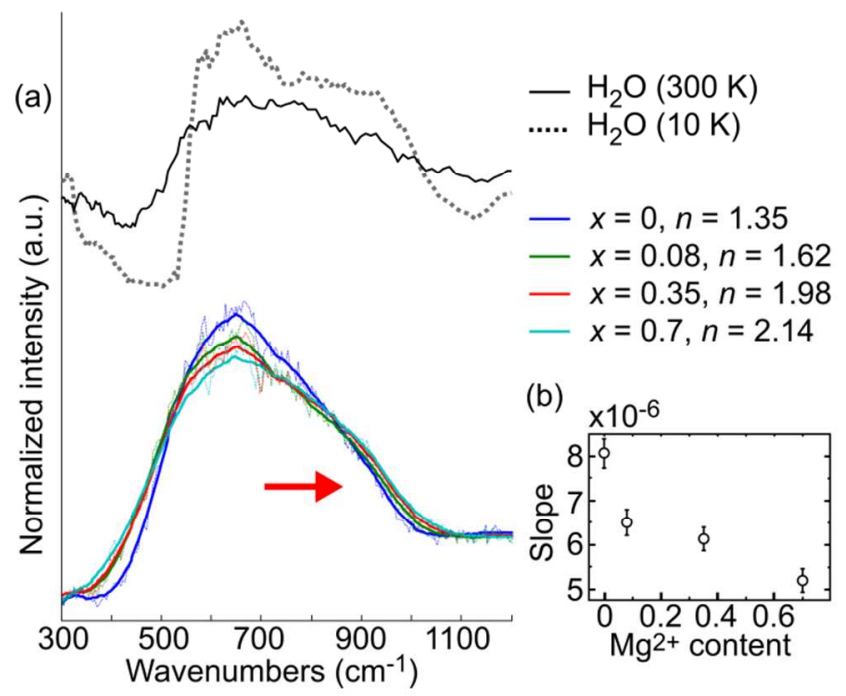

Figure 1. (a) Librational region of the generalized vibrational density of states (GDOS) for water at 300 and $10 \mathrm{~K}$ (top) and $\mathrm{Mg}^{2+}$-doped ACC at $10 \mathrm{~K}$ (bottom) for $x=0$ (pure ACC), 0.08 , 0.35 , and 0.7 . The intensity is normalized to the unity within the 300 to $1200 \mathrm{~cm}^{-1}$ range. Thick lines represent data smoothed over 20 points for better visualization. The red arrow indicates the frequency range where the stiffening of $\mathrm{H}$-bonds is displayed (see text). (b) Slope of the librational edge from 400 to $600 \mathrm{~cm}^{-1}$ as a function of $\mathrm{Mg}^{2+}$ content.

At higher frequencies (from $\sim 600$ to $1100 \mathrm{~cm}^{-1}$, the socalled librational region), the positions and the intensities of the modes are sensitive to the local H-bond environment. ${ }^{47,48}$ Our DFT calculations successfully show that the structural water in monohydrocalcite exhibits well-defined librational spectra with discrete modes comparable to experimental data (Supporting Figure S4). Despite the lack of discrete modes in amorphous carbonates, we observed a relative increase of the higher frequency modes above $850 \mathrm{~cm}^{-1}$ that is proportional to the $\mathrm{Mg}^{2+} / \mathrm{Ca}^{2+}$ ratio (red arrow in Figure 1a bottom), pointing to an increase in the stiffness of the H-bonds. This observation was further confirmed by DFT simulations of hydrated $\mathrm{Ca}^{2+}$ and $\mathrm{Mg}^{2+}$ ions (Supporting Figure $\mathrm{S} 5$ ), thus strongly supporting the existence of stiffer H-bonding networks when $\mathrm{Mg}^{2+}$ is present. This stiffening can be associated to the high dehydration energy of $\mathrm{Mg}^{2+}$, reflected in the frequency of exchange events in the hydration water, which is three orders of magnitude lower than that of $\mathrm{Ca}^{2+}{ }^{29}$ Other type of divalent ion inclusions, such as $\mathrm{Sr}^{2+}$ or $\mathrm{Ba}^{2+}$, are also known in biogenic ACC. ${ }^{49,50}$ However, these ions did not demonstrate any stiffening effect (Supporting Figure S6), which could be due to their larger ionic size and the subsequent lability of water from their first hydration shell. These results therefore highlight the fact that $\mathrm{Mg}^{2+}$ acts as a stronger center of charge, contributing to the formation of localized and stiff H-bonding networks within amorphous carbonates. To further understand the role of 
$\mathrm{Mg}^{2+}$ in association to the stiffening, we determined the thermal stability of water present in the amorphous samples by identifying, in a purely operational way, two types of water, namely mobile and rigid (see Supporting Figure S3 for the detailed methodology). The former represents adsorbed or surface-bound water, whereas the latter can be regarded as structural water. $^{22}$ Figure 2 shows that, besides the anticipated increase of the total water content with increasing $\mathrm{Mg}^{2+} / \mathrm{Ca}^{2+}$ ratio, the fraction of rigid, structural water increases more significantly than mobile water as $\mathrm{Mg}^{2+} / \mathrm{Ca}^{2+}$ ratio increases. This non-negligible presence of structural water can be related to the local stiffening of $\mathrm{H}$-bonding induced by $\mathrm{Mg}^{2+}$. This in turn may indeed have contributed to the kinetic persistence of $\mathrm{Mg}^{2+}$-doped ACC (Supporting Figure S7) which is also in agreement with previous studies both in air and in solution. $^{38,51,52}$

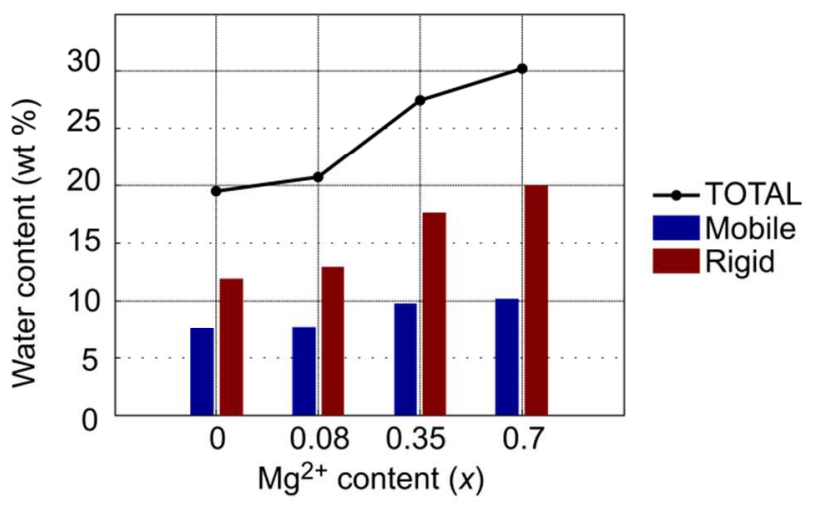

Figure 2. Water contents in $\mathrm{Mg}^{2+}$-doped ACC for $x=0$ (pure ACC), 0.08, 0.35, and 0.7 and the distribution into mobile and rigid structural components, determined by TGA analysis.

The H-bond network comprises three different types of interactions: 1) $\mathrm{H}_{2} \mathrm{O}-\mathrm{H}_{2} \mathrm{O}$, 2) $\mathrm{H}_{2} \mathrm{O}-\mathrm{Ca}^{2+} / \mathrm{Mg}^{2+}$ and 3) $\mathrm{H}_{2} \mathrm{O}-\mathrm{CO}_{3}{ }^{2-}$. A plausible hypothesis to explain the enhanced structural disorder in the H-bonding network and the increased kinetic persistence of $\mathrm{Mg}^{2+}$-bearing amorphous carbonates as compared to pure ACC is a stiffening of $\mathrm{H}$-bond network in the close vicinity to $\mathrm{Mg}^{2+}$ (H-bond type 2 ). Whether this stiffening extends to the whole water network needs further discussion. A study by Sen et al., ${ }^{53}$ as well as some previous studies, ${ }^{54}$ demonstrated that there is a strong $\mathrm{H}$-bond interaction between the water molecules and the carbonate units, with the latter experiencing significant structural distortion that is uniformly distributed within ACC, which in turn hinders crystallization due to steric frustration. The authors added, however, that this distortion is independent of the presence of $\mathrm{Mg}^{2+}$, except that the increased amount of water molecules could allow a closer packing, and thus a stronger interaction of water molecules and carbonate units. Others studies, on the other hand, suggested that the lower molar volume of $\mathrm{Mg}^{2+}$ as compared to $\mathrm{Ca}^{2+}$ introduces structural distortions (strain) in the host $\mathrm{Ca}$ $\mathrm{CO}_{3}$ structures that effectively inhibit crystallization or formation of periodic ordering, independently of cation-hydration effects. $^{55,56} \mathrm{Mg}$-doped amorphous calcium carbonates have been described as forming a solid-solution kind of structure in the region of low $\mathrm{Mg}$ content $(x<0.5)$, and an heterogeneous mixture of Mg-rich and Ca-rich domains at high $\mathrm{Mg}$ content $(x$ $>0.5) .{ }^{38}$ The fact that $\mathrm{Mg}^{2+}$-doped carbonates show an en- hanced kinetic persistence in the whole compositional range (Supporting Figure S7), and that no partial crystallization or segregation of $\mathrm{CaCO}_{3}$ is observed, leads to the hypothesis that the $\mathrm{Mg}^{2+}$-induced stiffening of the $\mathrm{H}$-bond network extends beyond the first $\mathrm{Mg}^{2+}$ hydration shell and can thus affect the H-bonding of type 1 and 3 .

Dynamics of structural relaxation. According to the classical nucleation theory, the rate of nucleation, $J_{\text {n, }}$ takes the form:

$$
J_{n}=A e^{-\frac{\Delta g}{K_{B} T}}
$$

with the pre-exponential term $A$ being the kinetic factor and the argument of the exponential function being the thermodynamic factor where $\Delta g_{\mathrm{n}}$ is the nucleation barrier (for details see Supporting Information section 3. Classical nucleation theory). The activated character of the nucleation process implies that not all attempts are necessarily successful in overcoming the energetic barrier and that the system will undergo a certain number of conformational or structural rearrangements before a crystal is nucleated within the amorphous matrix. The frequency of structural rearrangements is actually one of the substantial factors dictating the kinetics of nucleation. The relaxation dynamics probed by XPCS provide a quantitative measurement on the frequency of these structural rearrangements and is therefore a good probe for the underlying dynamics. Our experimental set-up covered small scattering angles corresponding to distances of $\sim 25$ to $630 \mathrm{~nm}$. The observed relaxation dynamics are therefore not probed at the atomistic-scale, yet they cover a range that corresponds to density fluctuations within individual particles of amorphous carbonates with a typical size range in the order of tens to hundreds of nanometers ${ }^{38,57}$. To this point, we would like to stress that preliminary XPCS measurements made at the atomistic-scale (i.e., high $q$ ) revealed extremely fast relaxation dynamics that were technically inaccessible with the 2D detector employed. This highly dynamic character of the amorphous carbonates contrasts with the dynamic regimes present in other amorphous and glassy systems such as covalent or metallic glasses, which exhibit relaxation times that are at least four orders of magnitude larger. ${ }^{58,59}$

Figure 3a shows a representative time-evolution of $g^{(2)}(\Delta t, q)$ for the pure ACC $(x=0)$ averaged every 33 minutes over the duration of a measurement. A significant slowing down of the relaxation dynamics - aging - was observed with time, and similar trends were found in all the other samples (Supporting Figure S8a). The aging nature is best demonstrated by the evolution of $\tau$ values (Figure $3 \mathrm{~b}$ ) and seemingly it could indicate the onset of crystallization. Strikingly, however, the corresponding time-evolution of the intensity profiles over the full $q$ range (i.e. static structure, $\mathrm{S}(q)$ ) displays no apparent change over time (Figure 3c). This surprising phenomenon was common in all the amorphous carbonates examined in the present study (Supporting Figure S8b). Additional test measurements were performed to verify and exclude the possibility of an artefact induced by the X-ray beam (Supporting Figure S9). The aging in our amorphous carbonates could instead be explained by the partial dehydration due to the active vacuum during the course of the measurements. Indeed, ex-situ TGA measurements performed on samples subject to the same vacuum conditions showed a very small dehydration, in the order of $1-2 \% \mathrm{w} / \mathrm{w}$ (Supporting Figure S10). Such minor dehydration may nevertheless suffice to trigger subtle struc- 
tural changes, imperceptible in the static structure factor, $\mathrm{S}(q)$, as pointed out by Giordano and Ruta. ${ }^{35}$ The authors demonstrated that structural change of metallic glasses as minor as $1 \%$ in the lattice parameter was responsible for significant changes in the relaxation dynamics (one order of magnitude). It is also to note that the interpretation proposed above is consistent with simulation results by Wallace et al. ${ }^{60}$ showing that the self-diffusion of $\mathrm{Ca}^{2+}$ within the amorphous structure decreases monotonically as the samples are dehydrated. (a)

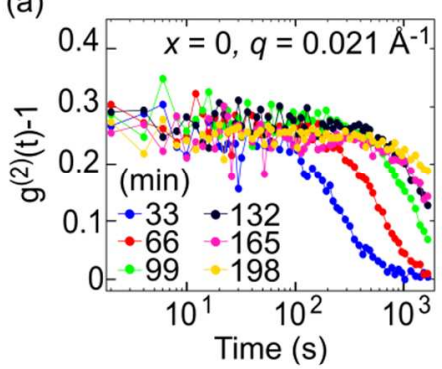

(c)

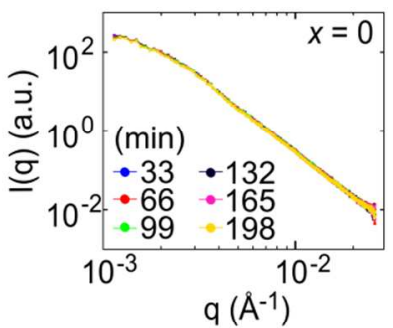

(b)

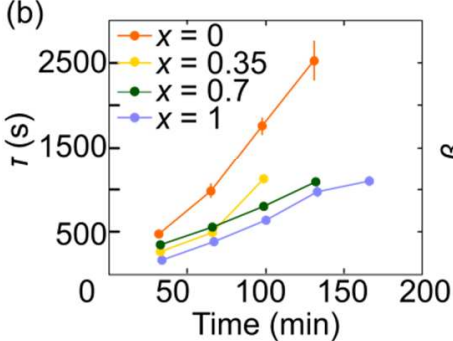

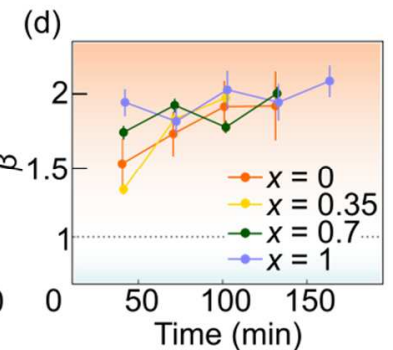

Figure 3. Aging dynamics. (a) Representative time-evolution of the intensity auto-correlation functions over the course of the measurement for ACC at $q=0.021 \AA^{-1}(d \sim 30.5 \mathrm{~nm})$. (b) Corresponding time-evolution of the intensity profile for ACC, i.e. static structure factor $\mathrm{S}(q)$, for the full $q$ range measured $(0.001 \leq$ $q \leq 0.023 \AA^{-1}$ ). (c) Relaxation time $\tau$ and (d) shape factor $\beta$ as a function of the experimental time for $\mathrm{Mg}^{2+}$-doped ACC at $x=0$ (pure ACC), 0.35, 0.7 and 1 (pure AMC). Note the sample $x=$ 0.35 was measured under slightly different conditions (see text).

The observed stronger H-bonding network induced by the presence of $\mathrm{Mg}^{2+}$, and the enhanced kinetic persistence of $\mathrm{Mg}^{2+}$-bearing amorphous carbonates, seemed to provide a strong suggestion that the $\mathrm{Mg}^{2+}$-bearing samples should exhibit slower relaxation dynamics than pure ACC. Remarkably, the XPCS data demonstrate the opposite trend as shown in Figure 4 and as summarized in Table 1. These data clearly demonstrate that the relaxation time $\tau$ is smaller for $\mathrm{Mg}^{2+}$ doped ACC. In other words, the number of structural rearrangements per unit of time $(1 / \tau)$ is higher for $\mathrm{Mg}^{2+}$-doped amorphous carbonates, with a non-linear proportionality to the $\mathrm{Mg}^{2+}$ and water contents (Figure $4 \mathrm{~b}$ ). In addition, the rate of aging reflects the persistence of $\mathrm{AMC}$ and $\mathrm{Mg}^{2+}$-bearing amorphous carbonates in comparison to that of ACC (Figure $3 b)$ : the time-evolution of the measured relaxation times $(\tau)$ shows that ACC displays the most dramatic changes, with a five-fold increase. $\mathrm{Mg}^{2+}$-bearing samples are thus less prone to age, a fact related to their stability and/or kinetic persistence as opposed to that of pure ACC.
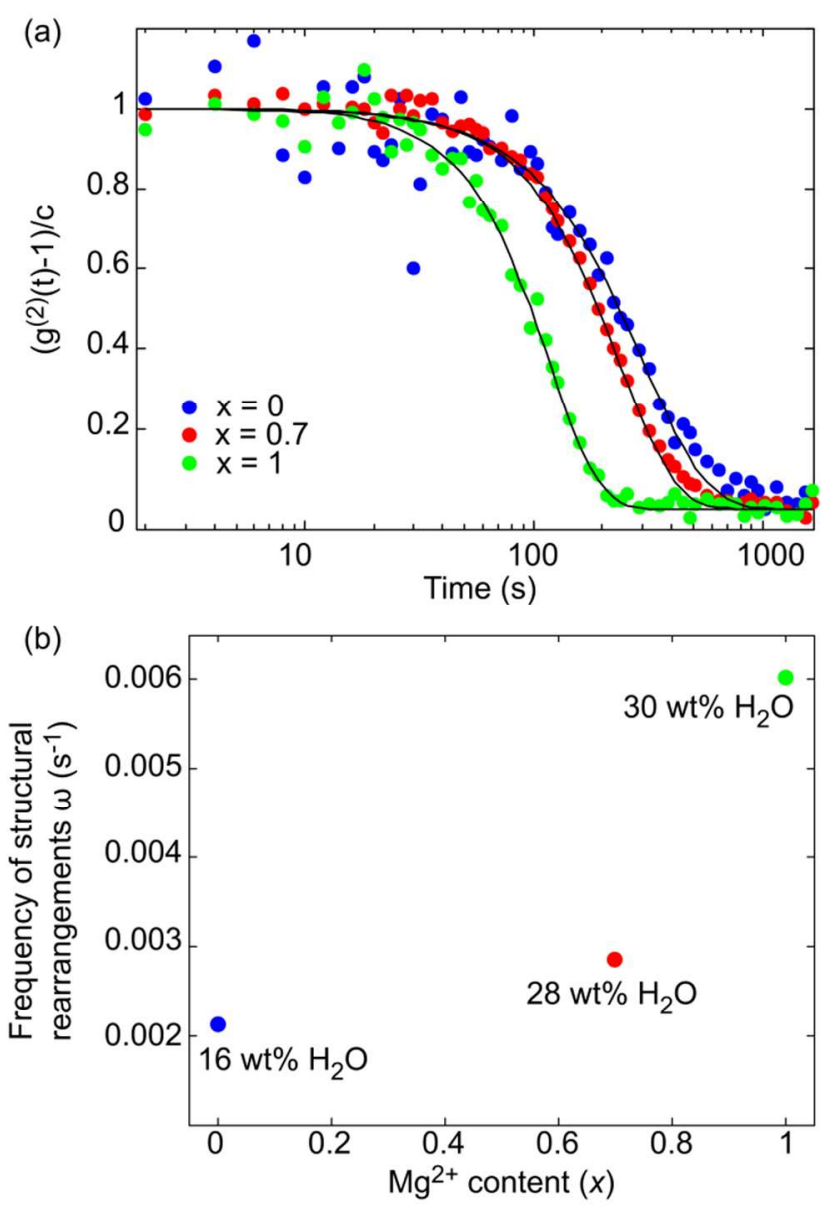

Figure 4. (a) Normalized intensity auto-correlation functions at $\mathrm{q}$ $=0.021 \AA^{-1}(d \sim 30.5 \mathrm{~nm})$ for $\mathrm{Ca}_{(1-x)} \mathrm{Mg}_{x} \mathrm{CO}_{3} n \mathrm{H}_{2} \mathrm{O}$ with $x=0,0.7$ and 1. Fitted data are shown in solid lines. (b) Frequency of structural rearrangement $(\omega=1 / \tau)$ as a function of $\mathrm{Mg}^{2+}$ and water contents.

Besides the aging phenomena and the unexpectedly dynamic character of the structural relaxation that is enhanced in presence of $\mathrm{Mg}^{2+}$, our data suggest that the nature of such relaxation seem to be subject to internal stresses. The first indicator is the compressed decay (shape factor $\beta>1$ ) of the autocorrelation functions, $g^{(2)}(\Delta t, q)$, in all of our amorphous samples, which, in addition, increases proportionally to the $\mathrm{Mg}^{2+}$ content (Table 1) and to the aging time (Figure 3d). The second indicator is the dependence of the relaxation time $\tau$ on $q$, which displays proportionality to $q^{-1}$ (Supporting Figure S11). This has previously been shown to be indicative of ballistic dynamics which can accompany $\beta>1$ for systems bearing internal stresses, ${ }^{61}$ in contrast to ideal Brownian diffusive systems (refer to Supporting Information section 3 for details). These results infer that: (i) the partial dehydration under active vacuum is accompanied by an increase in the stress within the hydrated amorphous solids; and (ii) that the presence of $\mathrm{Mg}^{2+}$ in the structure is what introduces internal stresses. As suggested by studies on metallic glasses ${ }^{62,63}$ and discussed earlier, the origin of the observed stress could be due to the structural distortion known in the $\mathrm{CO}_{3}{ }^{2-}$ unit coordinated to water molecules as discussed earlier. The stiff $\mathrm{H}$ - 
bonding network imposed by $\mathrm{Mg}^{2+}$ could possibly be an additional factor leading to internal stress.

Table 1. Fitted parameters $(\tau, \beta)$ obtained from the intensity auto-correlation functions

\begin{tabular}{llll}
\hline $\mathrm{Ca}_{1-x} \mathrm{Mg}_{x} \mathrm{CO}_{3} \cdot n \mathrm{H}_{2} \mathrm{O}$ & & \\
$x$ & $n$ & $\tau(\mathrm{s})$ & $\beta$ \\
\hline 0 & 1.08 & $470 \pm 39$ & $1.54 \pm 0.17$ \\
0.7 & 1.96 & $350 \pm 6$ & $1.76 \pm 0.05$ \\
1 & 1.99 & $166 \pm 4$ & $1.97 \pm 0.09$ \\
\hline
\end{tabular}

Given the isotropic character of the amorphous carbonates, and the lack of recognizable higher order or hierarchical structural arrangements, the dynamic behavior observed here at the nanoscale can be extrapolated to the atomic scale. Relaxation times can therefore be interpreted as the lifetimes of a given structural configuration. A link can then be established between the number of structural rearrangements per unit of time $(\omega)$ and the crystallization kinetics. Under these considerations, let us closely examine the kinetic term $A$ in eq. (3). The idea behind is that in a (metastable) amorphous system, when the nucleation barrier is high enough compared to the thermal energy, the metastable state of the system contains thermal fluctuations that are well described by a Boltzmann distribution. Sometimes, some of these fluctuations will reach and overcome the critical size, leading to crystallization. The kinetic term $A$ may adopt various forms depending on the nature of the nucleation event (see Supporting Information section $3)^{64,65}$, though qualitatively speaking, it includes 1) the number of trials (formation of critical clusters) to overcome the nucleation barrier per unit of time $(\omega), 2)$ the probability that these trials are successful or not (Zeldovich factor, $Z$ ), and 3) the number of sites per unit of volume where the clusters can be formed $\left(C_{0}\right){ }^{65}$ In nucleation processes within disordered materials, such as the case here, the 'coherent diffusivity' measured by XPCS is an adequate representative of the physical mechanisms underlying the crystallization process, as it integrates both the self-diffusion of molecular or ionic species within the solid and their relative diffusivity (refer to Supporting Information section 3). Here, it is to note that whether water acts as a mediator (via partial or interfacial dissolution reprecipitation) or not (in a solid state reaction) is of no relevance, since both processes would lead to structural rearrangements that are probed with XPCS. A direct proportionality can thus be established between the nucleation rate, $J_{n}$, and the XPCS-determined number of structural re-arrangements per unit of time $(\omega)$ :

$$
J_{n} \propto A \mathrm{e}^{-\frac{\Delta g}{K_{\mathrm{B}} T}} \propto \omega Z C_{0} \mathrm{e}^{-\frac{\Delta g}{K_{\mathrm{B}} T}}
$$

This rationalization in eq. (4) can be used to interpret our experimental data in terms of the different physically meaningful factors. Given the slower nucleation rate observed in $\mathrm{Mg}^{2+}$-bearing systems, their faster dynamics (higher $\omega$ ) have to be compensated by the other factors, provided that the $\mathrm{Mg}^{2+}$-bearing ACC are less prone to crystallize. The strong $\mathrm{H}-$ bonding network induced by the presence of $\mathrm{Mg}^{2+}$ is hypothesized as the main reason behind this increased kinetic persistence. The internal stress revealed by XPCS measurements and the stiffening of $\mathrm{H}$-bonds shown by IINS are probably two manifestations of the same phenomenon: an enhancement of the H-bond stiffness of the water network that results in a decrease of the success rate of nucleation trials $(Z)$, and thus lowers the crystallization rate of $\mathrm{Mg}^{2+}$-bearing amorphous carbonates.

\section{Conclusion}

The unique combination of coherent X-ray and incoherent neutron scattering techniques has provided a novel way to describe the mechanism by which the inclusion of $\mathrm{Mg}^{2+}$ increases the kinetic persistence of amorphous carbonates. Neutron scattering experiments probing the dynamics of $\mathrm{H}$ bonding network have shown a stiffening of the intermolecular bonds. Unexpectedly, this stiffening is accompanied by an increase of the coherent diffusivity within the $\mathrm{Mg}^{2+}$-bearing amorphous carbonate samples (translated as enhanced structural rearrangements), and to an internally stressed structure, probably due to the molecular deformations induced by the strained $\mathrm{Mg}^{2+}$ environment. It is proposed that the addition of the highly bonded $\mathrm{Mg}^{2+}$ hydration water to the amorphous systems causes local stresses that impede crystallization. Future experiments will be devoted to ascertain the universality of this stabilization mechanism, using other types of additives.

\section{ASSOCIATED CONTENT}

\section{Supporting Information.}

The Supporting Information is available free of charge at http://pubs.acs.org.XXX

\section{AUTHOR INFORMATION}

\section{Corresponding Author}

*Alex.Fernandez-Martinez@univ-grenoble-alpes.fr

\section{Present Address}

${ }^{1}$ Ayumi Koishi

Department of Civil and Environmental Engineering, Princeton University, Princeton, New Jersey 08544, United States

\section{ACKNOWLEDGMENT}

Gaston Garbarino and Alexander Van Driessche are thanked for constructive discussions and experimental help. A.K. acknowledges the French Ministry of Higher Education, Research and Innovation for the doctoral fellowship. A.F.-M. acknowledges the CNRS program EC2CO for financial support. TGA was funded from Labex OSUG@2020 (investissements d'avenir; ANR10 LABX56). The ESRF and ILL facilities are thanked for beamtime allocation.

\section{REFERENCES}

(1) Mass, T.; Giuffre, A. J.; Sun, C.-Y.; Stifler, C. A.; Frazier, M. J.; Neder, M.; Tamura, N.; Stan, C. V.; Marcus, M. A.; Gilbert, P. U. P. A. Amorphous Calcium Carbonate Particles Form Coral Skeletons. Proc. Natl. Acad. Sci. 2017, 114 (37), E7670-E7678. Beniash, E.; Aizenberg, J.; Addadi, L.; Weiner, S. Amorphous Calcium Carbonate Transforms into Calcite during Sea Urchin Larval Spicule Growth. Proc. R. Soc. B Biol. Sci. 1997, 264 (1380), 461-465.

(3) Politi, Y.; Arad, T.; Klein, E.; Weiner, S.; Addadi, L. Sea Urchin Spine Calcite Forms via a Transient Amorphous Calcium Carbonate Phase. Science 2004, 306 (12), 1161-1164.

(4) Weiss, I. M.; Tuross, N.; Addadi, L.; Weiner, S. Mollusc Larval Shell Formation: Amorphous Calcium Carbonate Is a Precursor Phase for Aragonite. J. Exp. Zool. 2002, 293 (5), 478-491. 
(5) Addadi, L.; Raz, S.; Weiner, S. Taking Advantage of Disorder: Amorphous Calcium Carbonate and Its Roles in Biomineralization. Adv. Mater. 2003, 15 (12), 959-970.

(6) Aizenberg, J. New Nanofabrication Strategies: Inspired by Biomineralization. MRS Bull. 2010, 35 (04), 323-330.

(7) De Yoreo, J. J.; Gilbert, P. U. P. A.; Sommerdijk, N. A. J. M.; Penn, R. L.; Whitelam, S.; Joester, D.; Zhang, H.; Rimer, J. D.; Navrotsky, A.; Banfield, J. F.; et al. Crystallization by Particle Attachment in Synthetic, Biogenic, and Geologic Environments. Science 2015, 349 (6247), aaa6760-1-aaa6760-9.

(8) Rodriguez-Blanco, J. D.; Shaw, S.; Benning, L. G. The Kinetics and Mechanisms of Amorphous Calcium Carbonate (ACC) Crystallization to Calcite, Viavaterite. Nanoscale 2011, 3 (1), 265-271.

(9) Weiner, S.; Levi-Kalisman, Y.; Raz, S.; Addadi, L. Biologically Formed Amorphous Calcium Carbonate. Connect. Tissue Res. 2003, 44 (Suppl 1), 214-218.

(10) Gotliv, B. A.; Addadi, L.; Weiner, S. Mollusk Shell Acidic Proteins: In Search of Individual Functions. ChemBioChem 2003, 4 (6), 522-529.

(11) Aizenberg, J.; Addadi, L.; Weiner, S.; Lambert, G. Stabilization of Amorphous Calcium Carbonate by Specialized Macromolecules in Biological and Synthetic Precipitates. Adv. Mater. 1996, 8 (3), 222-226.

(12) Whittaker, M. L.; Dove, P. M.; Joester, D. Nucleation on Surfaces and in Confinement. MRS Bull. 2016, 41 (05), 388392.

(13) Loste, E.; Park, R. J.; Warren, J.; Meldrum, F. C. Precipitation of Calcium Carbonate in Confinement. Adv. Funct. Mater. 2004, 14 (12), 1211-1220.

(14) Raiteri, P.; Gale, J. D. Water Is the Key to Nonclassical Nucleation of Amorphous Calcium Carbonate. J. Am. Chem. Soc. 2010, 132 (49), 17623-17634.

(15) Giuffre, A. J.; Gagnon, A. C.; De Yoreo, J. J.; Dove, P. M. Isotopic Tracer Evidence for the Amorphous Calcium Carbonate to Calcite Transformation by Dissolution-reprecipitation. Geochim. Cosmochim. Acta 2015, 165, 407-417.

(16) Purgstaller, B.; Mavromatis, V.; Immenhauser, A.; Dietzel, M. Transformation of Mg-Bearing Amorphous Calcium Carbonate to Mg-Calcite - In Situ Monitoring. Geochim. Cosmochim. Acta 2016, 174, 180-195.

(17) Lippmann, F. Crystal Chemistry of Sedimentary Carbonate Minerals. In Sedimentary Carbonate Minerals. Minerals, Rocks and Inorganic Materials (Monograph Series of Theoretical and Experimental Studies), vol. 6.; Springer: Berlin, Heidelberg, 1973; pp 5-96.

(18) Berner, R. A. The Role of Magnesium in the Crystal Growth of Calcite and Aragonite from Sea Water. Geochim. Cosmochim. Acta 1975, 39, 489-504.

(19) Davis, K. J.; Dove, P. M.; Yoreo, J. J. De. The Role of Mg2+ as an Impurity in Calcite Growth. 2000, 290 (5494), 1134-1137.

(20) Politi, Y.; Metzler, R. A.; Abrecht, M.; Gilbert, B.; Wilt, F. H.; Sagi, I.; Addadi, L.; Weiner, S.; Gilbert, P. U. P. A. Transformation Mechanism of Amorphous Calcium Carbonate into Calcite in the Sea Urchin Larval Spicule. Proc. Natl. Acad. Sci. U. S. A. 2008, 105 (45), 17362-17366.

(21) Beniash, E.; Addadi, L.; Weiner, S. Cellular Control over Spicule Formation in Sea Urchin Embryos: A Structural Approach. J. Struct. Biol. 1999, 125 (1), 50-62.

(22) Ihli, J.; Wong, W. C.; Noel, E. H.; Kim, Y.-Y.; Kulak, A. N.; Christenson, H. K.; Duer, M. J.; Meldrum, F. C. Dehydration and Crystallization of Amorphous Calcium Carbonate in Solution and in Air. Nat. Commun. 2014, 5.

(23) Konrad, F.; Gallien, F.; Gerard, D. E.; Dietzel, M. Transformation of Amorphous Calcium Carbonate in Air. Cryst. Growth Des. 2016, 16 (11), 6310-6317.

(24) Raz, S.; Hamilton, P. C.; Wilt, F. H.; Weiner, S.; Addadi, L. The Transient Phase of Amorphous Calcium Carbonate in Sea Urchin Larval Spicules: The Involvement of Proteins and Magnesium Ions in Its Formation and Stabilization. Adv. Funct. Mater. 2003, 13 (6), 480-486.

(25) Schmidt, M. P.; Ilott, A. J.; Phillips, B. L.; Reeder, R. J. Structural Changes upon Dehydration of Amorphous Calcium Carbonate. Cryst. Growth Des. 2014, 14 (3), 938-951.

(26) Michel, F. M.; MacDonald, J.; Feng, J.; Phillips, B. L.; Ehm, L.;
Tarabrella, C.; Parise, J. B.; Reeder, R. J. Structural Characteristics of Synthetic Amorphous Calcium Carbonate. Chem. Mater. 2008, 20 (14), 4720-4728.

(27) Radha, A. V; Navrotsky, A. Direct Experimental Measurement of Water Interaction Energetics in Amorphous Carbonates $\mathrm{MCO} 3(\mathrm{M}=\mathrm{Ca}, \mathrm{Mn}$, and $\mathrm{Mg})$ and Implications for Carbonate Crystal Growth. Cryst. Growth Des. 2015, 15, 70-78.

(28) Reeder, R. J.; Tang, Y.; Schmidt, M. P.; Kubista, L. M.; Cowan, D. F.; Phillips, B. L. Characterization of Structure in Biogenic Amorphous Calcium Carbonate: Pair Distribution Function and Nuclear Magnetic Resonance Studies of Lobster Gastrolith. Cryst. Growth Des. 2013, 13 (5), 1905-1914.

(29) Di Tommaso, D.; De Leeuw, N. H. First Principles Simulations of the Structural and Dynamical Properties of Hydrated Metal Ions $\mathrm{Me} 2+$ and Solvated Metal Carbonates $(\mathrm{Me}=\mathrm{Ca}, \mathrm{Mg}$, and Sr). In Crystal Growth and Design; 2010; Vol. 10, pp 42924302.

(30) Raiteri, P.; Demichelis, R.; Gale, J. D. Thermodynamically Consistent Force Field for Molecular Dynamics Simulations of Alkaline-Earth Carbonates and Their Aqueous Speciation. $J$. Phys. Chem. C 2015, 119 (43), 24447-24458.

(31) Kerisit, S.; Parker, S. C. Free Energy of Adsorption of Water and Metal Ions on the $\{1014\}$ Calcite Surface. J. Am. Chem. Soc. 2004, 126 (32), 10152-10161.

(32) Ockwig, N. W.; Cygan, R. T.; Hartl, M. A.; Daemen, L. L.; Nenoff, T. M. Incoherent Inelastic Neutron Scattering Studies of Nanoconfined Water in Clinoptilolite and Heulandite Zeolites. J. Phys. Chem. C 2008, 112 (35), 13629-13634.

(33) Ding, M.; Watkins, E. B.; Hartl, M.; Daemen, L. L. Water Signatures and Their Thermal Stability in Bedded Salt for Nuclear Waste Storage: An Incoherent Inelastic Neutron Spectroscopy Study. Environ. Sci. Technol. Lett. 2015, 150928143203004.

(34) Ockwig, N. W.; Greathouse, J. A.; Durkin, J. S.; Cygan, R. T.; Daemen, L. L.; Nenoff, T. M. Nanoconfined Water in Magnesium-Rich 2 : 1 Phyllosilicates. JACS 2009, No. 6, 81558162 .

(35) Giordano, V. M.; Ruta, B. Unveiling the Structural Arrangements Responsible for the Atomic Dynamics in Metallic Glasses during Physical Aging. Nat. Commun. 2015, 7, 1-8.

(36) Dierker, S. B.; Pindak, R.; Fleming, R. M.; Robinson, I. K.; Berman, L. X-Ray Photon-Correlation Spectroscopy Study of Brownian-Motion of Gold Colloids in Glycerol. Phys. Rev. Lett. 1995, 75 (3), 449-452.

(37) Radha, A. V.; Forbes, T. Z.; Killian, C. E.; Gilbert, P. U. P. A.; Navrotsky, A. Transformation and Crystallization Energetics of Synthetic and Biogenic Amorphous Calcium Carbonate. Proc. Natl. Acad. Sci. U. S. A. 2010, 107, 16438-16443.

(38) Radha, A. V.; Fernandez-Martinez, A.; Hu, Y.; Jun, Y.-S.; Waychunas, G. A.; Navrotsky, A. Energetic and Structural Studies of Amorphous Ca1-xMgxCO3 $\cdot \mathrm{nH} 2 \mathrm{O} \quad(0 \leqslant \mathrm{x} \leqslant 1)$. Geochim. Cosmochim. Acta 2012, 90, 83-95.

(39) Koga, N.; Nakagoe, Y.; Tanaka, H. Crystallization of Amorphous Calcium Carbonate. Thermochim. Acta 1998, 318 (1-2), 239-244.

(40) Kojima, Y.; Kawanobe, A.; Yasue, T.; Arai, Y. Synthesis of Amorphous Calcium Carbonate and Its Crystallization. $J$. Ceram. Soc. Japan 1993, 101 (1178), 1145-1152.

(41) Nebel, H.; Neumann, M.; Mayer, C.; Epple, M. On the Structure of Amorphous Calcium CarbonatesA Detailed Study by SolidState NMR Spectroscopy. Inorg. Chem. 2008, 47 (17), 78747879.

(42) Han, K.-L.; Zhao, G.-J. Hydrogen Bonding and Transfer in the Excited State; John Wiley \& Sons Ltd, 2011.

(43) Ivanov, A.; Jimenéz-Ruiz, M.; Kulda, J. IN1-Lagrange-the New ILL Instrument to Explore Vibration Dynamics of Complex Materials. J. Phys. Conf. Ser. 2014, 554.

(44) Bikondoa, O. On the Use of Two-Time Correlation Functions for X-Ray Photon Correlation Spectroscopy Data Analysis. $J$. Appl. Crystallogr. 2017, 50 (Pt 2), 357-368.

(45) Ruta, B.; Baldi, G.; Monaco, G.; Chushkin, Y. Compressed Correlation Functions and Fast Aging Dynamics in Metallic Glasses. J. Chem. Phys. 2013, 138 (5).

(46) Evenson, Z.; Ruta, B.; Hechler, S.; Stolpe, M.; Pineda, E.; Gallino, I.; Busch, R. X-Ray Photon Correlation Spectroscopy 
Reveals Intermittent Aging Dynamics in a Metallic Glass. Phys. Rev. Lett. 2015, 115 (17).

(47) Li, J.-C.; Londono, D.; Ross, D. K.; Finney, J. L.; Bennington, S. M.; Taylor, a D. Inelastic Incoherent Neutron Scattering Study of Ice Ih, II, IX, V and VI in the Region from 50-500 MeV. J. Phys. Condens. Matter 1999, 4 (9), 2109-2116.

(48) Li, J.-C.; Londono, J. D.; Ross, D. K.; Finney, J. L.; Tomkinson, J.; Sherman, W. F. An Inelastic Incoherent Neutron Scattering Study of Ice II, IX, V, and VI-in the Range. J. Chem. Phys. 1991, 94 (May).

(49) Couradeau, E.; Benzerara, K.; Gerard, E.; Moreira, D.; Bernard, S.; Brown, G. E.; Lopez-Garcia, P. An Early-Branching Microbialite Cyanobacterium Forms Intracellular Carbonates. Science 2012, 336 (October 2015), 459-462.

(50) Cam, N.; Georgelin, T.; Jaber, M.; Lambert, J.-F.; Benzerara, K. In Vitro Synthesis of Amorphous Mg-, Ca-, Sr- and BaCarbonates: What Do We Learn about Intracellular Calcification by Cyanobacteria? Geochim. Cosmochim. Acta 2015, 161, 3649.

(51) Loste, E.; Wilson, R. M.; Seshadri, R.; Meldrum, F. C. The Role of Magnesium in Stabilising Amorphous Calcium Carbonate and Controlling Calcite Morphologies. J. Cryst. Growth 2003 , 254 (1-2), 206-218.

(52) Rodriguez-Blanco, J. D.; Shaw, S.; Bots, P.; Roncal-Herrero, T; Benning, L. G. The Role of $\mathrm{PH}$ and $\mathrm{Mg}$ on the Stability and Crystallization of Amorphous Calcium Carbonate. J. Alloys Compd. 2012, 536, S477-S479.

(53) Sen, S.; Kaseman, D. C.; Colas, B.; Jacob, D. E.; Clark, S. M. Hydrogen Bonding Induced Distortion of $\mathrm{CO} 3$ Units and Kinetic Stabilization of Amorphous Calcium Carbonate: Results from 2D 13 C NMR Spectroscopy. Phys. Chem. Chem. Phys. 2016, 7, 689-702.

(54) Saharay, M.; Yazaydin, A. O.; Kirkpatrick, R. J. DehydrationInduced Amorphous Phases of Calcium Carbonate. J. Phys. Chem. B 2013, 117 (12), 3328-3336.

(55) Xu, J.; Yan, C.; Zhang, F.; Konishi, H.; Xu, H.; Teng, H. H. Testing the Cation-Hydration Effect on the Crystallization of Ca-Mg-CO3 Systems. Proc. Natl. Acad. Sci. U. S. A. 2013, 110 (44), 17750-17755.

(56) Politi, Y.; Batchelor, D. R.; Zaslansky, P.; Chmelka, B. F.; Weaver, J. C.; Sagi, I.; Weiner, S.; Addadi, L. Role of
Magnesium Ion in the Stabilization of Biogenic Amorphous Calcium Carbonate: A Structure-Function Investigation. Chem. Mater. 2010, 22 (1), 161-166.

(57) Zou, Z.; Bertinetti, L.; Politi, Y.; Jensen, A. C. S.; Weiner, S.; Addadi, L.; Fratzl, P.; Habraken, W. J. E. M. Opposite Particle Size Effect on Amorphous Calcium Carbonate Crystallization in Water and during Heating in Air. Chem. Mater. 2015, 27 (12), 4237-4246.

(58) Horbach, J.; Kob, W.; Binder, K. Dynamics of Sodium in Sodium Disilicate: Channel Relaxation and Sodium Diffusion. Phys. Rev. Lett. 2002, 88 (12).

(59) Ruta, B.; Chushkin, Y.; Monaco, G.; Cipelletti, L.; Pineda, E.; Bruna, P.; Giordano, V. M.; Gonzalez-Silveira, M. AtomicScale Relaxation Dynamics and Aging in a Metallic Glass Probed by X-Ray Photon Correlation Spectroscopy. Phys. Rev. Lett. 2012, 109.

(60) Wallace, A. F.; Hedges, L. O.; Fernandez-Martinez, A.; Raiteri, P.; Gale, J. D.; Waychunas, G. A.; Whitelam, S.; Banfield, J. F.; De Yoreo, J. J. Microscopic Evidence for Liquid-Liquid Separation in Supersaturated CaCO3 Solutions. Science 2013 341, 885-889.

(61) Ferrero, E. E.; Martens, K.; Barrat, J. L. Relaxation in Yield Stress Systems through Elastically Interacting Activated Events. Phys. Rev. Lett. 2014, 113 (24), 1-5.

(62) Shen, Y. T.; Kim, T. H.; Gangopadhyay, A. K.; Kelton, K. F. Icosahedral Order, Frustration, and the Glass Transition: Evidence from Time-Dependent Nucleation and Supercooled Liquid Structure Studies. Phys. Rev. Lett. 2009, 102 (5), 6-9.

(63) Li, W.; Bei, H.; Tong, Y.; Dmowski, W.; Gao, Y. F. Structural Heterogeneity Induced Plasticity in Bulk Metallic Glasses: From Well-Relaxed Fragile Glass to Metal-like Behavior. Appl. Phys. Lett. 2013, 103 (17).

(64) Clouet, E. Modeling of Nucleation Processes. In ASM Handbook Vol. 22A, Fundamentals of Modeling for Metals Processing; Furrer, D. U., Semiatin, S. L., Eds.; 2010; pp 203 219.

(65) Kashchiev, D. Nucleation: Basic Theory with Applications; Butterworth-Heinemann: Oxford, 2000. 
Authors are required to submit a graphic entry for the Table of Contents (TOC) that, in conjunction with the manuscript title, should give the reader a representative idea of one of the following: A key structure, reaction, equation, concept, or theorem, etc., that is discussed in the manuscript. Consult the journal's Instructions for Authors for TOC graphic specifications.

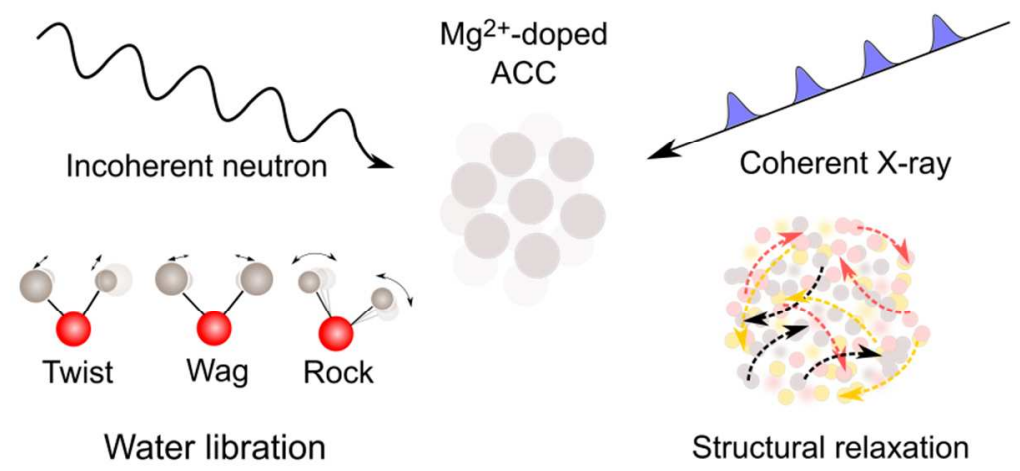

